\title{
Información presupuestaria y derechos económicos, sociales y culturales: lo procedimental es normativo
}

\section{Economic, social and cultural rights and budget information: Procedural is normative}

\author{
Diego de la Mora Maurer \\ Fundar, México
}

Licenciado en Ciencias Políticas y Relaciones Internacionales por el Centro de Investigación y Docencia Económicas (CIDE, México). Actualmente estudia la maestría en Derechos Humanos y Democracia en la Facultad Latinoamericana de Ciencias Sociales (FLACSO), sede México. Desde 2008 trabaja en Fundar, Centro de Análisis e Investigación en el Área de presupuestos y políticas públicas, donde analiza la relación entre los presupuestos públicos, la transparencia y la rendición de cuentas.

diego@fundar.org.mx

\section{Miguel Pulido Jiménez \\ Fundar, México}

\begin{abstract}
Abogado del Instituto Tecnológico y de estudios superiores de Monterrey (México). Cursó la maestría en Derechos Humanos y Democracia por la Facultad Latinoamericana de Ciencias Sociales (FLACSO), sede México y el diploma de postítulo en Justicia Transicional y Procesos de Democratización en el Centro de Derechos Humanos de la Facultad de Derecho de la Universidad de Chile. Desde 2009 es Director Ejecutivo de Fundar, Centro de Análisis e Investigación. Sus áreas de especialización son los derechos humanos, la transparencia y los sistemas de rendición de cuentas comparados.
\end{abstract}

miguel@fundar.org.mx

\section{RESUMEN}

Los derechos económicos, sociales y culturales tradicionalmente fueron vistos como programáticos y han tenido un desarrollo marginal respecto de otros derechos humanos. Esto ha llevado a un escaso desarrollo de técnicas de supervisión de cumplimiento de las obligaciones del Estado en relación a estos derechos. En este artículo, los autores explican que a través del análisis del comportamiento del Estado manifestado en sus presupuestos públicos, es posible determinar si existe una violación a estos derechos. Dado que el presupuesto determina las posibilidades materiales de avanzar en el cumplimiento de los derechos, sostienen que su contenido trasciende la esfera de lo político y lo programático y se transforma en un asunto de orden jurídico. Ejemplificando este enfoque desde la experiencia mexicana, los autores concluyen su análisis proponiendo referentes prácticos para la gestión presupuestaria con enfoque de derechos humanos.

Palabras clave: Derechos económicos, sociales y culturales - presupuestos públicos - indicadores de cumplimiento - exigibilidad - progresividad.

\begin{abstract}
Economic, social and cultural rights have been seen traditionally as programmatic and have had little development compared to other human rights. This has hampered the development of supervision techniques for State compliance with this type of rights. This article suggests that it is possible to conclude rights violation by analysing public budgets. Given that budgets determine State compliance possibilities with rights, the article supports the idea that budgetary contents transcends both political and programmatic spheres becoming part of the legal order. Using the Mexican experience as a model, the article proposes concrete measures for a human rights oriented budget management.
\end{abstract}

Key words: Economic, social and cultural rights public budget - compliance index - enforceability - progressivity. 


\section{Introducción}

Los derechos son construcciones sociales y, en estos términos, no es fácil abstraerlos de posiciones ideológicas o de matices políticos. De hecho, durante su desarrollo (y/o su construcción) también son receptivos de influencias de carácter cultural y económica, tanto por los tiempos en los que se construyen, como por las sociedades en que se desarrollan. En el caso de los derechos humanos, la dogmática ha desarrollado la categoría de derechos económicos, sociales y culturales (DESC), que por diversas razones han tenido un desarrollo marginal y diferenciado de otras categorías, por oposición y contraste, podemos decir que principalmente de los denominados derechos civiles y políticos.

Autores como Courtis y Abramovich ${ }^{1}$ han desenmascarado una de las críticas que con mayor frecuencia se realiza contra los DESC, aquella que plantea que no son derechos en el mismo sentido o con la misma validez que los derechos civiles y políticos. Ferrajoli, Pisarello y otros autores también han abordado esta cuestión ${ }^{2}$. El punto neurálgico de la crítica a los DESC es que se les cataloga como derechos programáticos, por lo que se los tilda de incompletos, por ejemplo, al no ser invocables frente a los jueces, y se pretende sostener que están dirigidos únicamente a guiar la acción de los poderes ejecutivos y legislativos. Quienes sostienen esta perspectiva les restan justamente el que sean derechos exigibles y justiciables, mirándolos ante todo, como establece Pisarello, como mandatos políticos, o si acaso, normas de efecto indirecto, mediato. Son presentados como derechos condicionados, cuya exigibilidad ante órganos jurisdiccionales aparece inevitablemente supeditada a la previa interposición legislativa y administrativa ${ }^{3}$. Pero entre los efectos de este tratamiento diferenciado no está solo la dimensión judicial; el desarrollo de instrumentos de medición relacionados directamente con la evaluación del grado de satisfacción de derechos es también un campo que presenta cierto rezago. La realización progresiva se ha confundido con la declaración de falta de elementos de objetivación o parámetros razonables de medición de su cumplimiento (y por tal razón, de su violación). Al considerarse pautas de orientación de la actuación del Estado, los grados de incumplimiento de los DESC no se entienden como desviaciones del mandato de garantizar normas jurídicas y, por tanto, a la evidencia generada no suele dársele una aproximación jurídica.

Sin embargo, al menos en las últimas dos décadas, se presentan cambios sustanciales en diversos campos tanto de estudio como de análisis. En las líneas que siguen presentamos diversos argumentos, metodologías y propuestas que se han desarrollado con el fin de contribuir al avance sustancial de respeto, goce y garantía de los DESC. Daremos especial atención a los argumentos que se construyen desde los instrumentos de medición del grado de avance en la satisfacción de los derechos y su vínculo con medidas de exigibilidad (directa o indirecta) usando la técnica presupuestaria. Nuestro punto central es que cierta evidencia sobre el comportamiento del Estado en realidad constituye un elemento de prueba para saber si ahí donde se presentan insatisfacciones de derechos se está ante una violación a los derechos humanos que debe ser revertida. Nuestra afirmación es que, desde esta perspectiva, la información presupuestaria trasciende lo procedimental y es de carácter normativo.

ABRAMOVICH, Víctor y COURTIS, Christian. Los derechos sociales como derechos exigibles. Madrid: Trotta, 2003.

Véase por ejemplo: FERRAJOLI, Luigi. Derecho y razón. Madrid: Trotta, 1995 y PISARELLO, Gerardo. "El Estado Social como Estado constitucional: mejores garantías, más democracia". En: ABRAMOVICH, Víctor (et. al.). Derechos Sociales, Instrucciones de Uso. México: Fontamara, 2003.

PISARELLO, Gerardo. El Estado social como Estado constitucional... op.cit, p.28. 


\section{Un método emergente para la vigencia de los DESC: la medición}

El desarrollo del conocimiento sobre las capacidades del Estado para garantizar los derechos humanos, ha avanzado a tal grado que hoy es posible analizar cuáles son las condiciones que permiten que la mayoría de las personas puedan satisfacer sus requerimientos vitales y tener una vida más digna. Algunas de esas condiciones, sino es que la mayoría, pueden ser provistas a partir de políticas y programas estatales que aseguren el acceso oportuno a bienes y servicios de calidad, a precios accesibles y de forma que se adapten a las prácticas culturales tanto de las personas como de las comunidades.

La evidencia muestra que para llevar los derechos humanos desde aspiraciones éticas plasmadas en leyes hasta su realización efectiva y puesta en práctica, el Estado requiere diseñar, implementar y evaluar un conjunto políticas públicas ${ }^{4}$. Las obligaciones consagradas en los instrumentos internacionales e incorporados en varias constituciones nacionales son un elemento de referencia para estos tres fines (diseño, implementación y evaluación de políticas). Existen una serie de principios que, primero, permiten orientar el quehacer estatal $y$, posteriormente, pueden servir para confrontar las políticas existentes con dichos principios y para fijar marcos para la elaboración e implementación de las políticas públicas ${ }^{5}$.

Hasta ahora, el avance de los derechos humanos se ha medido a partir de indicadores diseñados para brindar información concreta "sobre el estado o la condición de un objeto, un acontecimiento, una actividad o un resultado" ${ }^{\prime}$. El valor de dichas mediciones, como se aprecia, es de tipo descriptivo. Sin embargo, en los últimos años, diversos actores involucrados en la defensa de los derechos humanos han avanzado en la generación y puesta a punto de distintos sets de indicadores que no solo permiten conocer el estado de situación respecto a ciertos derechos, sino ponderar las acciones emprendidas por los Estados y determinar si dichas acciones soportan un estándar de evaluación. Entre estos indicadores encontramos los de tipo presupuestario que dan cuenta de la priorización en la asignación de recursos y develan cuál es el alcance del conjunto de acciones emprendidas. La existencia de cierta información presupuestaria desagregada permite identificar y calcular los emprendimientos por parte de los gobiernos de cara al avance de los distintos derechos.

Desde la academia y las organizaciones de la sociedad civil, se han construido instrumentos que permiten monitorear estos procesos. Entre estas formas de monitoreo y seguimiento de los derechos destaca el marco de análisis "OPERA" del Centro de Derechos Económicos, Sociales y Culturales (CESR por sus siglas en inglés). Se trata de una herramienta que mide resultados (outcomes), esfuerzos políticos (policy efforts), recursos (resourses) y valoración (assestment) para visualizar lo que hacen los Estados para materializar los derechos. Este marco propone la medición de las obligaciones esenciales, de la igualdad y la no discriminación, la realización progresiva y la no retrogresión, la utilización del máximo de recursos disponibles, entre otras.

Otra herramienta análoga es el "Índice de Cumplimiento de los Derechos Económicos y Sociales"

Oficina del Alto Comisionado de Naciones Unidas para Derechos Humanos (ACNUDH). Indicadores para Derechos Humanos: guía para la medición y la aplicación. HR/PUB/12/5, 2012, capítulo 3.

PISARELLO, Gerardo. El Estado social como Estado constitucional... op.cit, p.44.

ACNUDH. Indicadores para Derechos Humanos... op.cit, p.19.

Center for Economic and Social Rights. OPERA Framework. Disponible [en línea] <http://www.cesr.org> [consulta: 24 de abril de 2014]. 
(SERF), elaborado por la Iniciativa para el Empoderamiento de los Derechos Económicos y Sociales $^{8}$, la cual mide el desempeño de los países y sus entidades subnacionales en el cumplimiento de los derechos económicos y sociales. Esta herramienta utiliza métodos econométricos para calcular índices por derechos (alimentación, salud, educación y vivienda) y a partir de ahí construir un índice de cumplimiento de las obligaciones esenciales.

Estas propuestas metodológicas requieren de la disponibilidad de distintas variables presupuestarias. OPERA propone indicadores de impacto, información desagregada por grupos de población, así como indicadores sobre el uso de los recursos públicos, entre otros. El SERF utiliza indicadores económicos disponibles como el PIB per cápita y los contrasta con indicadores de derechos sociales y económicos que también están disponibles como: número de niños/as desnutridos/as, uso de anticonceptivos, tasa de supervivencia de niños/as menores a cinco años, entre otros.

\section{El presupuesto público como acción de Estado}

Durante la última década, cada vez más países latinoamericanos han otorgado jerarquía constitucional a los tratados internacionales de derechos humanos ${ }^{9}$. Esta internalización del Derecho Internacional de los Derechos Humanos (DIDH) implica cambios fundamentales en la organización de los Estados de la región, tales como adecuar el diseño institucional de sus gobiernos para que puedan cumplir con las obligaciones de derechos humanos contraídas a través de la ratificación de los tratados internacionales.

A pesar de que los Estados latinoamericanos han modificado, en casi todos los casos, sus regímenes políticos para construir sistemas democráticos que han permitido avanzar en el cumplimiento de los derechos civiles y políticos; en el caso de los DESC parece no existir el mismo avance: prueba de ello es que América Latina sigue siendo una de las regiones más pobres y la más desigual del mundo ${ }^{10}$.

El instrumento jurídico de mayor referencia respecto a las obligaciones de los Estados para el cumplimiento de los DESC es el Pacto Internacional de los Derechos Económicos, Sociales y Culturales (PIDESC) ${ }^{11}$. El artículo 2 del PIDESC establece que los Estados parte deben adoptar medidas para avanzar progresivamente y sin discriminación en la plena efectividad de los derechos reconocidos por dicho pacto, utilizando el máximo de los recursos de los que dispongan. Precisamente, entre las medidas que se tendrían que adoptar, están las económicas y técnicas. Desde esta perspectiva, las políticas relacionadas con los ingresos y egresos del gobierno son garantías de tipo operativo para salvaguardar derechos.

8

Economic and Social Rights Empowerment Initiative. Index of Economic and Social Rights Fulfillment, 2011. Disponible [en línea] <http://www.serfindex.org> [consulta: 24 de abril de 2014].

9 FERRER MAC-GREGOR. "Interpretación conforme y control difuso de convencionalidad. El nuevo paradigma para el juez mexicano". En: CARBONELL, Miguel y SALAZAR, Pedro (Coords.) La reforma constitucional de derechos humanos: un nuevo paradigma. México: Instituto de Investigaciones Jurídicas UNAM, 2011, pp.355-356. the Caribbean. Social Panorama of Latin America 2012. Santiago: ECLAC, 2013. Americana de Derechos Económicos, Sociales y Culturales o "Protocolo de San Salvador". Dicho protocolo recupera las obligaciones de tomar medidas, especialmente económicas y técnicas, utilizar el máximo uso de los recursos disponibles de manera progresiva (artículo 1) y de garantizar los DESC sin discriminación (artículo 3). 
Nos concentramos solo en los presupuestos públicos en tanto son las herramientas con las que cuentan los Estados para administrar y gestionar los recursos públicos y en ellos se establece el programa del gasto y la forma, cantidad y destino de los recursos públicos. En el caso de México, por ejemplo, ahí se prevé la distribución general a los tres ámbitos de gobierno y las distintas instituciones de los poderes de la Unión (ejecutivo, judicial, legislativo) además de organismos autónomos y empresas estatales ${ }^{12}$.

Al reflejar las prioridades de los gobiernos, los presupuestos son una especie de radiografía o mapa para conocer si un Estado ha realizado las acciones a las que jurídicamente está obligado. Nuestra afirmación es que dado que el presupuesto determina las posibilidades materiales de avanzar en el cumplimiento de los derechos (y nuestro punto de interés es el caso de los derechos sociales) al establecer la cantidad de recursos que el Estado podrá utilizar para su realización, su contenido trasciende la esfera de lo político y lo programático y se convierte en un asunto de orden jurídico. En estos términos, no existe diferencia técnica entre un Estado que omite medidas para erradicar la tortura y uno que no dispone adecuadamente de sus recursos para asegurar el acceso de la mayor cantidad de personas a los servicios de salud, por ejemplo.

La información presupuestaria no solo permite conocer la correlación de fuerzas entre los grupos que se disputan los recursos públicos. Además de esta forma política de entender el presupuesto, hay otra en la que su contenido es evidencia que puede ser usada para exigir al Estado cambios en la forma que actúa. El régimen hacendario, junto con el sistema legal, es el vehículo idóneo para la salvaguarda de los derechos humanos. De ahí que su gestión esté también sujeta a la obligación de respetar el contenido de los tratados internacionales y otras normas que prevean obligaciones de comportamiento a los Estados.

Este cambio de paradigma implica que los Estados transformen la forma en la que proyectan, aprueban y fiscalizan sus presupuestos. En el caso mexicano, la información presupuestaria disponible está organizada para gestionar los recursos orientados a objetivos que tradicionalmente están basados en un esquema de facultades y atribuciones de las dependencias públicas y no necesariamente para la realización de derechos. El enfoque de derechos en materia presupuestaria gira este enfoque hacia uno orientado a los resultados e impactos de las acciones gubernamentales en la calidad de vida de las personas, lo que implica reconocer que los derechos también pueden ser violados por las interacciones que ocurren dentro del Estado y que le impidan poder entregar bienes y servicios.

\section{Cambios al enfoque presupuestario: lo procedimental es normativo}

La formulación de políticas públicas con perspectiva de derechos humanos es más que una agenda programática, se trata de la expresión técnica de una obligación jurídica. En el caso mexicano, por ejemplo, como signante del PIDESC, ¿qué significa este enfoque normativo? En principio, la incorporación de las obligaciones de dicho Pacto como eje rector en todo el ciclo de presupuestación. El enfoque de derechos humanos se convierte entonces en un estándar que regula la actuación presupuestaria y permite identificar si quienes diseñan, implementan y evalúan las políticas y los presupuestos públicos tuvieron en cuenta en su actuación las prioridades del Estado y su relación con los derechos humanos.

12 Secretaría de Hacienda y Crédito Público (SHCP). Presupuesto Ciudadano 2013. México. Disponible [en línea] $<$ http://www.transparenciapresupuestaria.gob.mx/ptp/ServletImagen?tipo=pdf\&idDoc=383> [consulta: 27 de abril de 2014]. 
La incorporación del enfoque de derechos al presupuesto ordena las funciones del Estado. A la luz de esta afirmación, la clasificación funcional (que responde a la pregunta ¿para qué se gasta?), da cuenta de las acciones realizadas para el avance de los derechos humanos reflejando posibles omisiones y por tanto violaciones directas a derechos jurídicamente tutelados. El núcleo central de este argumento es que la información contenida en las herramientas administrativas (proyectos de presupuestos, presupuestos, cuentas públicas, matrices de marco lógico, entre otras) con las que cuenta el gobierno federal mexicano reflejan prima facie si ante la situación que guardan los derechos humanos se están tomando las medidas pertinentes. Conocer cuánto y cómo se gastan los recursos destinados a los grupos de población en situación de vulnerabilidad, por ejemplo, visibiliza el grado de atención y servicios que el Estado les está proveyendo $y$, permite diagnosticar si los recursos son suficientes para atender sus requerimientos. Es decir, la clasificación presupuestaria permite conocer si se están gastando los recursos con dicho fin. En caso negativo, esta información debe ser usada por distintas instancias para reconducir el ejercicio de los recursos para mejorar la calidad y la dignidad de la vida de las personas.

A nivel federal, en México, el presupuesto público se clasifica de cinco formas: administrativa, funcional programática, económica, por fuente de financiamiento y geográfica. Estas clasificaciones dan información relevante sobre quién, en qué, para qué y en dónde gasta los recursos ${ }^{13}$. Las 3 clasificaciones más utilizadas son: la administrativa, la económica y la funcional/programática que explica qué es lo que se busca lograr con el gasto (en términos de programas de salud, educación, vivienda, seguridad pública, legislación, entre otras). En muchas ocasiones más de una secretaría o unidad responsable ejercen la misma función ${ }^{14}$.

La clasificación funcional/programática agrupa el gasto con base en las actividades que por disposición legal, le corresponden a los ejecutores de gasto de acuerdo con los resultados que se proponen alcanzar en términos de finalidades (FI), funciones (FN), subfunciones (SF), actividades institucionales $(\mathrm{Al})$, programas presupuestarios (PP), proyectos, actividades, indicadores, objetivos y metas. En un escenario ideal, dicha clasificación permite conocer y evaluar, de acuerdo con el artículo 134 de la Constitución, la eficiencia, eficacia, transparencia, honradez y economía con la que se utiliza el gasto público en cada una de las etapas del proceso presupuestario.

En su conjunto, toda esta información permite conocer si el gasto está atendiendo la satisfacción de los DESC. Sin embargo, conviene hacer una aclaración. El uso de la información presupuestaria desde una perspectiva normativa requiere trascender la relación lineal que solo vincule la nomenclatura de la clasificación presupuestaria con los derechos humanos. Es decir, no es un asunto de denominación, sino de funcionalidad del gasto. Por ejemplo, el gobierno mexicano ha clasificado los derechos humanos desde la lógica administrativa y como parte de sus funciones de justicia y gobierno, es decir, desde una perspectiva legal acotada que no reconoce la provisión de bienes y servicios como parte de la garantía de los DESC. De ahí que para identificar si el Estado está usando el máximo de recursos disponibles, la fuente de evidencia no es aquella clasificación presupuestaria que mencione la palabra derechos humanos, sino la identificación del conjunto de acciones orientadas a su satisfacción.

De hecho, del conjunto de información presupuestaria, son el nivel de función (FN) y la ac-

13 SHCP. Manual de Programación y Presupuestación 2014. México, p.14. Disponible [en línea] <http://www.shcp. gob.mx/EGRESOS/PEF/programacion/programacion_2014/manual_pyp/manual_PyP_2014.pdf> [consulta: 24 de abril de 2014].

14 En el caso del PEF 2014 la clasificación geográfica solo hace referencia a las entidades federativas donde se ejerce el gasto y a la clave de cartera de los programas y proyectos de inversión. 
tividad institucional $(\mathrm{Al})$, dos de las fuentes de información que permiten conocer con mayor detalle si el gobierno mexicano está gestionando los recursos públicos para cumplir con sus obligaciones de derechos humanos. Las Al están conformadas por las acciones sustantivas de las distintas instancias del gobierno para cumplir los objetivos y metas de los programas. De esta forma es posible conocer, por ejemplo, si las acciones de salud en beneficio de una población específica están recibiendo recursos suficientes.

¿Cuáles son los efectos de esta perspectiva para una agenda de exigibilidad de los DESC? Un ejemplo puede sintetizar nuestra perspectiva: en tanto el presupuesto es un mandato con fuerza legal de ejecución de gasto público, grupos afectados por la falta de asignación de recursos suficientes para el ejercicio y goce de los derechos sociales estarían en condiciones de enderezar un reclamo -incluso judicial- en el que desafíen al Estado a demostrar que exploró todas las alternativas posibles para la satisfacción de sus derechos. Entre las consecuencias de la falta de asignación están la corrección de la conducta (por una orden judicial) o -cuando se trate de la etapa de aprobación del presupuesto- por la intervención del Poder Legislativo. Esto resulta al contrastar el hecho jurídico (la asignación de fondos) con el estándar de protección de derechos humanos previsto en el PIDESC y las instrucciones de comportamiento dirigidas a los Estados (máximo uso de recursos disponibles, no regresión, progresividad y no discriminación).

\section{Referentes prácticos para la gestión presupuestaria con enfoque de derechos humanos}

En cumplimiento de las obligaciones derivadas del PIDESC los gobiernos deben movilizar todos los recursos posibles al interior del país y el gasto debe ser eficiente y eficaz. Lo anterior significa que los recursos asignados para el cumplimiento de los DESC no pueden ser ejercidos para tareas o fines distintos al cumplimiento de dichos derechos. Algunas expresiones prácticas de este enfoque son las siguientes:

- Proporción de aumentos presupuestarios: En principio, el avance en el cumplimiento de derechos requiere el uso de recursos económicos suficientes. En casos como el mexicano, en el que, por ejemplo, amplios sectores de la población carecen de acceso a servicios de salud, anualmente se requiere destinar importantes recursos presupuestarios para brindar cobertura médica y atención hospitalaria a las personas. La negativa a asignar mayores recursos, en este caso, para no constituir un desvío de la obligación prevista en el PIDESC debe venir acompañada de explicaciones razonables y de justificaciones con valor jurídico que demuestren cómo la atención de la salud de las personas puede ser lograda sin la disposición de mayores recursos financieros. Las explicaciones sobre mejoras en las adquisiciones, economías y modernización administrativa son esenciales para estos fines.

- Justificación de las regresiones en tiempos de crisis: Si bien los Estados están obligados a usar los recursos disponibles, esto no significa que baste con alegar la indisponibilidad de recursos por razones de orden económico. En los tiempos de crisis, los Estados deben demostrar la cancelación de gastos no esenciales para la vigencia o cumplimiento de derechos y qué tipo de medidas y políticas contracíclicas serán tomadas para revertir las regresiones, en los casos cuya necesidad haya sido demostrada. Las disminuciones presupuestarias constituyen presunciones con validez jurídica de la afectación a derechos, por tal razón, el conjunto de medidas que las fundamenten deben incluir en su estructura argumentos sólidos que justifiquen no solo porqué los derechos serán temporalmente afectados, sino cuál es el conjunto de medidas que revertirán esta situación. 
- Aumentos proporcionales a DESC respecto a los incrementos globales y eliminación de gastos superfluos: Los gobiernos están obligados a avanzar progresivamente, sin retrocesos, hacia el cumplimiento de los DESC. La realización progresiva debe entenderse como mejoras continuas en la realización de los derechos. Esto implica aumentos presupuestarios que incorporen la inflación y medidas de racionalización de gasto privilegiando la satisfacción de derechos por sobre otras actividades institucionales o funciones que no guardan relación directa con la vigencia de derechos. En el caso de México, entre muchos otros gastos, podrían verse afectadas las partidas destinadas a la autopromoción de la imagen de los gobernantes, compensaciones salariales o el gasto en funciones duplicadas entre distintas secretarías de Estado.

- No discriminación: Para atenuar los efectos de la discriminación, los Estados pueden tomar acciones positivas temporales o permanentes. Para contrarrestar la discriminación sistémica, puede ser indispensable la asignación de recursos adicionales a la realización de los derechos de los grupos discriminados. Fallar en eliminar tratos diferenciados para distintos grupos, con base en insuficiencia de recursos, es inaceptable, a menos que el Estado demuestre haber usado el máximo de recursos disponibles.

\section{Conclusión}

El análisis del presupuesto público constituye evidencia sobre las prioridades reales de los gobiernos y sus políticas públicas. Esta información puede ser usada para participar activamente en la discusión sobre la distribución de los recursos públicos, así como para identificar la fuente de diversas problemáticas en el goce y disfrute de los DESC. Al contrastar las obligaciones que surgen del DIDH al presupuesto, es posible identificar si efectivamente los Estados están usando el máximo de recursos disponibles, si emprenden medidas de progresividad y no regresión, y si rigen su esquema de presupuestación en esquemas de no discriminación.

El conjunto de instrumentos de información presupuestaria constituyen evidencia contundente para identificar las brechas en el cumplimiento de los derechos de la población y de grupos históricamente discriminados. Las inequidades en el acceso a los derechos son condiciones susceptibles de corrección. Para ello se requiere el establecimiento de metas de corto, mediano y largo plazo para la realización progresiva de los derechos. En suma, el monitoreo de los fondos destinados a las políticas públicas trasciende un enfoque estrictamente de administración pública o gestión gubernamental y se convierte en un mecanismo propicio para medir el cumplimiento de los derechos humanos. Cuando la presupuestación se realice fuera de un enfoque de derechos, los Estados no están mal administrando recursos, están violando derechos. 